\title{
1 Unbiased detection of CRISPR off-targets in vivo using DISCOVER-Seq
}

2

3 Beeke Wienert ${ }^{1,2,3 \#}$, Stacia K Wyman ${ }^{1 \#}$, Christopher D Richardson ${ }^{1,2}$, Charles D Yeh ${ }^{1,2,4}$, Pinar

4 Akcakaya $^{5}$, Michelle J Porritt ${ }^{5}$, Michaela Morlock ${ }^{5}$, Jonathan T Vu ${ }^{1}$, Katelynn R Kazane ${ }^{1,2}$,

5 Hannah L Watry ${ }^{1,3}$, Luke M Judge ${ }^{3,6}$, Bruce R Conklin ${ }^{3,7}$, Marcello Maresca ${ }^{5}$ and Jacob E Corn M,2, $^{1,}$

6

$7{ }^{1}$ Innovative Genomics Institute, University of California, Berkeley, Berkeley, California, USA.

$8{ }^{2}$ Department of Molecular and Cell Biology, University of California, Berkeley, Berkeley,

9 California, USA.

$10{ }^{3}$ Gladstone Institutes, San Francisco, California, USA.

$11{ }^{4}$ Current address: Department of Biology, ETH Zurich, Switzerland.

$12{ }^{5}$ Discovery Biology, Discovery Sciences, IMED Biotech Unit, AstraZeneca, Gothenburg,

13 Sweden.

$14{ }^{6}$ Department of Pediatrics, UCSF, San Francisco, California, USA.

$15{ }^{7}$ Departments of Medicine, Ophthalmology \& Pharmacology, UCSF, San Francisco, California,

16 USA.

17 \# the authors contributed equally to this work

18 *corresponding author: jacob.corn@biol.ethz.ch 


\section{Abstract}

22 Genome editing using nucleases such as CRISPR-Cas induces programmable DNA damage at a

23 target genomic site but can also affect off-target sites. Here, we develop a powerful, sensitive assay

24 for the unbiased identification of off-target sites that we term DISCOVER-Seq. This approach

25 takes advantage of the recruitment of endogenous DNA repair factors for genome-wide

26 identification of Cas-induced double-strand breaks. One such factor, MRE11, is recruited precisely

27 to double-strand breaks, enabling molecular characterization of nuclease cut sites with single-base

28 resolution. DISCOVER-Seq detects off-targets in cellular models and in vivo upon adenoviral gene

29 editing of mouse livers, paving the way for real-time off-target discovery during therapeutic gene

30 editing. DISCOVER-Seq is furthermore applicable to multiple types of Cas nucleases and provides

31 an unprecedented view of events that precede repair of the affected sites.

\section{Introduction}

33 CRISPR-Cas (clustered regularly interspaced short palindromic repeats; CRISPR-associated)

34 genome editing holds great promise for therapeutic applications. CRISPR-Cas nucleases make

35 double-strand breaks (DSBs) at the intended target site but can also introduce unwanted mutations

36 at off-target sites within the genome. To bring CRISPR into the clinic, accurate characterization

37 of on- and off-target nuclease activity in any target cell population is critical (1).

39 A variety of in silico $(2-4)$, in vitro $(5-7)$, and cell-based assays $(8,9)$ have been developed to 40 predict or find CRISPR off-targets. While each of these orthogonal methods have their particular 
41 strengths, they also have certain weaknesses. Naïve prediction algorithms are for the most part

42 based on sequence similarity and currently have limited predictive power with very high false-

43 positive rates (10). Assays that induce DSBs in vitro, such as Digenome-Seq (5), CIRCLE-Seq (6)

44 and SITE-Seq (7), have high sensitivity but dramatically under- or overestimate the number of

45 target sites that are actually modified in cellular models or in vivo (11). Nuclease concentration

46 within the cell (7), delivery method (ribonucleoprotein (RNP) vs. plasmid) $(7,12,13)$ as well as

47 more complex cellular properties such as chromatin accessibility $(14,15)$ have been shown to

48 significantly affect editing outcomes and are generally missed by in vitro off-target assays. Cellular

49 assays, such as GUIDE-Seq (8), test nuclease cutting in a cellular context but rely on the integration

50 of an exogenous DNA oligo that is inefficient in primary cells and not applicable in vivo.

51 Furthermore, the co-transfection of additional exogenous DNA would not be used in human

52 therapy and may affect overall editing outcomes (16). Hybrid methods determine potential editing

53 sites in vitro followed by exhaustive testing in vivo via amplicon sequencing, but require testing

54 of hundreds to thousands of candidate sites with high false-positive rates (11). There remains a

55 pressing need for unbiased methods that characterize sites of genome editing in situ in human cells

56 and animal models without requiring extensive pre-treatment or introduction of additional editing

57 reagents beyond those used in gene editing therapies.

58 Here, we asked whether the recruitment of DNA repair proteins can be developed into a generally

59 applicable assay for the unbiased identification of sites of genome editing. Because the pathways

60 involved in DSB repair are broadly conserved among metazoans, such an approach is potentially

61 useful in a wide variety of contexts. After determining the recruitment kinetics of several repair

62 factors, we developed a powerful assay for the unbiased identification of off-target gene editing in

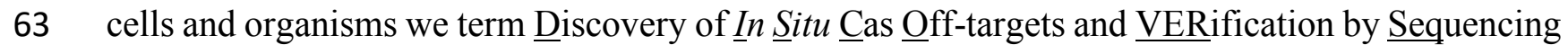


64 (DISCOVER-Seq). This approach involves chromatin immunoprecipitation followed by

65 sequencing (ChIP-Seq) of MRE11, followed by a custom computational pipeline called BLunt

66 END FindER (BLENDER). We find that MRE11 is broadly expressed across tissues and recruited

67 very early to a DSB with such precision that we are able to identify molecular characteristics of

68 nuclease cut sites with single nucleotide resolution. We demonstrate that DISCOVER-Seq reliably

69 detects off-target editing from a variety of guide RNAs, multiple Cas nucleases, in human and

70 mouse cells, and can even determine tissue and locus specificity during adenovirus-mediated in

71 vivo gene editing of mice.

\section{Results}

\section{Distinct binding characteristics of DNA repair proteins at Cas-induced DSBs}

74 While ChIP-Seq for catalytically inactive Cas9 nuclease has been explored to find off-targets (17,

75 18), this approach is plagued by many false positives because Cas9 binds far more sites than it

76 actually cleaves $(19,20)$. However, assembly of DNA repair proteins at Cas target sites indicates

77 that DNA damage has occurred, and by identifying DNA sequences that are bound by DNA repair

78 machinery one might theoretically map nuclease-induced sites of DNA damage genome-wide.

79 Several DNA repair proteins produce distinct microscopic foci at the locations of DSBs induced

80 by radiation or UV light (21). However, the molecular behavior of these factors at Cas nuclease-

81 induced break has not been studied in detail. We asked how various repair factors assemble at

82 Cas9-induced DSBs and if we could use this information to identify Cas9 cut sites in an unbiased

83 fashion. We began by performing ChIP-Seq experiments in K562 cells that were nucleofected with

84 a Cas9-RNP targeting VEGFA (Fig. 1A). We selected a panel of proteins involved in DNA repair 
85 of DSBs: Ser193-phosphorylated histone 2AX ( $\gamma \mathrm{H} 2 \mathrm{AX}), \mathrm{XRCC6} / \mathrm{Ku} 70, \mathrm{FANCD} 2$ and the three components of the MRN complex: MRE11, RAD50 and NBS1. We observed that all six DNA

87 repair proteins assembled at the targeted genomic location, but their binding patterns across the

88 site were highly distinctive (Fig. 1B). Both $\gamma \mathrm{H} 2 \mathrm{AX}$ and FANCD2 produced a broad peak centered 89 at the target site kilobases $(\mathrm{kb})$ to megabases $(\mathrm{mb})$ in length. Chromatin within $1-2 \mathrm{~kb}$ of the DSB 90 showed reduced occupancy by $\gamma \mathrm{H} 2 \mathrm{AX}$, consistent with previous reports $(22,23)$. Ku70 and 91 members of the MRN complex showed a defined narrow peak at the cut site, consistent with their 92 known roles in end processing (24-26) (Fig. 1C).

\section{The dynamics of DNA repair proteins at Cas9-induced DSBs}

94 To investigate how the binding of repair factors coincides with the formation of genetic insertions

95 and deletions (indels, the finished product of DNA repair) at the target site, we performed ChIP-

96 quantitative PCR (ChIP-qPCR) time course experiments using K562 cells edited with a Cas9-RNP

97 targeting the hemoglobin beta $(H B B)$ gene. We monitored both indel formation and binding of

$98 \gamma \mathrm{H} 2 \mathrm{AX}, \mathrm{FANCD} 2$ and MRE11 to the $H B B$ target and a previously described off-target site $(O T 1)$

$99(27,28)$ (Fig. 1D, Fig. S1 and Table S1). The presence of FANCD2 and $\gamma \mathrm{H} 2 \mathrm{AX}$ at the cut site 100 increased over time concurrently with the formation of indels, but MRE11 binding peaked at 12

101 hours post nucleofection and reduced as indels formed. These data suggest that MRE11 binds to the ends of a DSB but then disassociates, whereas FANCD2 and $\gamma \mathrm{H} 2 \mathrm{AX}$ may persist even as indels are forming at a site. MRE11 is therefore a good candidate to detect sites of Cas cleavage, since its action is immediate to the break both in space and time. 
ubiquitously expressed. We therefore tested protein expression levels of DNA repair proteins in a panel of human cell lines from diverse origins. Furthermore, we tested cross-reactivity of the antibodies used for ChIP-Seq between human and mouse, since an ideal off-target discovery method would be equally applicable not just in human cells but also in mice that are commonly used as a model organism for therapeutic editing. Of all proteins tested, only MRE11 showed high expression in all tested cell lines and was also recognized by an antibody that cross-reacts with murine Mre11 (Fig. 1E).

MRE11 ChIP-Seq provides single-nucleotide resolution of Cas-induced DSB

We examined the ChIP-Seq reads associated with each repair protein at the VEGFA target site and read pairs that start or end precisely at the cut site (e.g. are "blunt"), indicating binding to the ends of a DSB. We also classified read pairs that "span" the cut site (Fig. 2A, left). We found that DNA bound by FANCD2 and $\gamma \mathrm{H} 2 \mathrm{AX}$ predominantly span the cut site, though $\gamma \mathrm{H} 2 \mathrm{AX}$ occupancy was highest outside the cut site, consistent with prior reports (22) (Fig. 1A). DNA bound by the MRN

121 complex or Ku70 was primarily blunt at the cut site (Fig. 2A, right). These data confirm the roles 122 of Ku70 and the MRN complex as early responders to DSBs after Cas9-induced DNA damage and 123 illustrate that DNA fragments pulled down by MRN/Ku70 ChIP contain unrepaired DSBs (21).

124 Strikingly, we found that $\sim 90 \%$ of the reads in MRE11 peaks were blunt at the Cas9-induced cut 125 site. Paired-end 100-mer Illumina sequencing reads from MRE11 ChIP-Seq begin randomly on 126 one side of the break, due to fragmentation during sonication, and then end exactly at the Cas9 cut 127 site (Fig. S2A). While the insert size between the two paired ends varies, sequencing always starts 128 at the cut end, and thus alignment of paired reads causes a stack of 100-mers to each end of the cut 
129 site causing a characteristic shape of MRE11 ChIP-Seq peaks (Fig. S2A). The blunt side of 130 MRE11 binding to a Cas9-induced DSB is in fact so precise that the exact nucleotide 131 corresponding to the in vitro identified Cas9 nuclease site is identifiable by examining an MRE11

132 peak (Fig. 2B). Precise identification of a Cas9 cut site was possible for both the on-target site of 133 a $V E G F A$ targeting guide RNA (gRNA), as well as 32 known off-target sites (8) (Fig. S2B). To 134 the best of our knowledge, this is the first molecular observation of Cas9's in vitro nuclease activity 135 in a cellular context.

136 While the MRE11 peak at a Cas9 DSB is blunt on the PAM side of the VEGFA on-target site, it is 137 much more resected on the non-PAM side and hence asymmetric (Fig. 2B, Fig. S2A-B). Since we 138 previously observed that the non-PAM side of a Cas9 DSB is released first in vitro (29), we asked 139 if MRE11 peak asymmetry is predictable based on the location of the PAM. We aggregated 140 MRE11 peaks from the VEGFA on-target and 15 known off-targets and binned them based on 141 whether the PAM is oriented in the sense or anti-sense direction (Fig. 2C). Strikingly, the 142 asymmetry of a Cas9-induced MRE11 peak depends upon on the PAM orientation, such that the 143 blunt reads accumulate on the PAM side and the resected reads accumulate on the non-PAM side 144 (Fig. 2C). These data suggest that either the PAM side of a Cas9-induced DSB is protected against 145 early resection by cellular nucleases (30) or that cutting by Cas9 itself is not as precise on the non146 PAM side (31), both of which are equally consistent with in vitro data. Our observation of 147 asymmetric resection data in living cells is also consistent with in vitro and cellular data that Cas9 148 holds on more tightly to the PAM side and protects it from enzymatic modification (Fig. S2C) 149 (29). It may be that the asymmetric nature of Cas9-induced break influences the highly stereotyped 150 indel spectra reported for Cas9 editing (32-34). 
151 While SpCas9 makes relatively blunt-ended cuts (31), other nucleases such as Cas12a (also known

152 as Cpf1) produce staggered DSBs with a four nucleotide 5'-overhang (35). However, these

153 digestion patterns are based on in vitro data and it has so far not been possible to directly visualize

154 Cas12a nuclease activity in cells. We asked if MRE11 binding could be used to reveal Cas12a

155 activity during editing by nucleofecting an Acidaminococcus sp. Cas12a (AsCas12a) RNP

156 targeting the DNMT1 gene into K562 cells and performed ChIP-Seq for MRE11 (Fig. 2D). Unlike

157 SpCas9 MRE11 peaks, the AsCas12a MRE11 peak was quite symmetric around the predicted cut

158 sites. Furthermore, the reads at the targeted site overlapped one another but were blunt in 159 characteristic locations, consistent with the production of an overhang. However, the cellular

160 pattern of AsCas12a was not strictly a defined 4 nucleotide overhang as suggested in vitro, but

161 instead is ambiguous between 4-6 nucleotides. There is some evidence of mixed cleavage by

162 AsCas12a from in vitro studies (35) and our results indicate that the molecular site of nuclease

163 activity by AsCas12a in cells is not as simple as previously assumed. Taken together, our results

164 show that MRE11 ChIP-Seq can characterize nuclease-induced DSBs at a molecular level for

165 multiple types of Cas nucleases.

\section{DISCOVER-Seq detects off-targets in human cells}

167 While performing editing with the VEGFA targeting RNP, we manually identified MRE11 peaks

168 at known off-target sites for this gRNA. We next asked if we could develop MRE11 ChIP-Seq

169 into an unbiased method to discover CRISPR-Cas9 off-targets. We term this approach Discovery

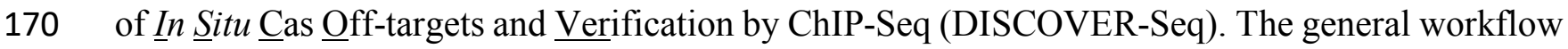

171 of DISCOVER-Seq is depicted in Fig. 3A and involves MRE11 ChIP-Seq followed by a 
173 signatures of Cas-induced breaks. BLENDER traverses genome-wide ChIP-Seq data, locating 174 stacks of reads and scoring each site based on the summed read ends within a window around the 175 cut site. BLENDER takes into account the unique features of MRE11 peaks, has several options 176 to optionally black-list known ChIP-Seq artifacts, references untreated control samples for 177 additional sensitivity, and filters based on protospacer identity for reduced false positives to thus 178 call even rare off-target events with great accuracy (Fig. S3).

179 We tested DISCOVER-Seq using the well-characterized, promiscuous 'VEGFA site 2' gRNA in 180 K562 cells (8). Unbiased DISCOVER-Seq identified 32 off-target sites for VEGFA_site2, which 181 we validated individually by amplicon next-generation sequencing (amplicon-NGS) four days 182 after nucleofection (Fig. 3B and Table S1). All off-targets that were identified by DISCOVER183 Seq were sites that have previously been described by GUIDE-Seq and were amplicon-NGS 184 validated with indel rates above background, with the least frequent site containing $0.29 \%$ indels 185 (Fig. 3B).

186 We also asked the converse, if there are major off-targets missed by DISCOVER-Seq but found 187 by GUIDE-Seq. We performed amplicon-NGS on all putative off-targets previously identified 188 with more than more than 100 GUIDE-Seq reads, but found that none of these sites had indel rates 189 above $1.2 \%$ (Table S1).

190 We further tested DISCOVER-Seq on a very specific guide targeting RNF2 with no known off191 targets, and a semi-specific guide targeting $H B B$ with two known off-targets. Again, we validated 192 all off-targets found with DISCOVER-Seq by amplicon-NGS (Fig. 3C-D). All sites identified with 193 DISCOVER-Seq showed indels above background and were thus bona fide off-targets. For the 194 RNF2 guide, DISCOVER-Seq found no sites other than the on-target site, confirming its 
specificity (8) (Fig. 3C). For the $H B B$ guide, DISCOVER-Seq successfully identified the two previously described off-target sites $(27,28)$ (Fig. 3D). Overall, these data show that DISCOVER-

197 Seq is very capable of unbiased in situ off-target detection in human cells. DISCOVER-Seq can

198

199

200

201

distinguish a specific gRNA from an unspecific gRNA and sensitively finds bona fide off-targets with no false positives (Fig. 3E). Furthermore, indel frequencies and DISCOVER scores were highly correlated suggesting that DISCOVER scores can be used to predict off-target mutation rates (Fig. 3E).

\section{Off-target discovery in murine cells}

As the MRE11 antibody used for DISCOVER-Seq is cross-reactive with the mouse protein, it can theoretically be used in a murine setting without modification of the protocol. Thus, we tested offtarget discovery with previously characterized promiscuous and highly specific gRNAs in murine cells. We edited the mouse skin melanoma cell line B16-F10 with two different Cas9-RNPs targeting the Pcsk9 gene and performed DISCOVER-Seq on these cells. The promiscuous Pcsk9 gRNA, "gP", has many closely matched sites in the mouse genome and thus many off-targets, while the specific gRNA, "gM", and has no off-targets (11). For gP edited cells, DISCOVER-Seq found 44 off-target sites in addition to the on-target Pcsk9 site (Fig. 4A and Fig. S4A). In this experiment, we found that DISCOVER-Seq was equally capable of identifying off-targets with the canonical SpCas9 PAM (NGG) and a known non-canonical PAM (NAG). For gM edited cells, DISCOVER-Seq exclusively found the on-target site (Fig. 4B). To validate the DISCOVER-Seq identified sites, we performed individual amplicon-NGS to determine indel frequencies after 4 days. We also performed amplicon-NGS at additional sites previously reported for the gP guide (11) (Fig. 4A-B and Table S2). All seven previously reported sites not identified by DISCOVER- 
217 Seq were very inefficiently edited, with indel frequencies below $0.43 \%$ and four of them had

218 indels below $0.1 \%$ (Table S2). DISCOVER scores and indel rates were correlated for both the gP

219 and $\mathrm{gP}+\mathrm{G}$ guide RNAs (Fig. S4A). We found that sites identified and validated with DISCOVER-

220 Seq had indel rates ranging from $0.1 \%$ to $66.1 \%$, showing that DISCOVER-Seq is applicable for

221 off-target detection in murine cell systems.

\section{Common additions to gRNAs alter off-target profiles}

223 Many research laboratories generate their gRNAs by in vitro transcription (in the case of RNP

224 editing) or transduce cells with plasmids or virus encoding gRNA. To ensure efficient transcription

225 from the T7 or U6 promoter, the protospacer must either begin with a 5' guanine (G), or an extra

2265 ' G must be added resulting in a 21-nt protospacer. Synthetic gRNAs do not require an extra 5'

$227 \mathrm{G}$ and can begin with any nucleotide. While the 5' $\mathrm{G}$ addition is relatively innocuous in terms of

228 on-target editing efficiencies, there is little data on how it affects off-target editing. Yet without

229 such knowledge, comparisons of off-target profiles between guides that contain an extra 5' G and

230 those that do not are potentially flawed.

231 The promiscuous Pcsk9 gP guide we used previously does not have a $\mathrm{G}$ in the first position but

232 has previously been used in mice via viral transduction (11) and thus was characterized with an

233 additional 5' G. We used DISCOVER-Seq to ask if adding an extra 5' G to Pcsk9 gP changes the

234 off-target profile of the gRNA. We edited B16-F10 cells with Cas9 and a gP guide containing an

235 extra 5' $\mathrm{G}(\mathrm{gP}+\mathrm{G})$ and performed DISCOVER-Seq. Comparing DISCOVER scores of gP and

$236 \mathrm{gP}+\mathrm{G}$, we found that the additional G markedly reduced off-target frequencies (Fig. 4C and Fig.

237 S4B). However, this reduction was not consistent across sites, and some off-target loci were still

238 detected with high frequency. Amplicon-NGS confirmed that on-target indel frequencies were 
239

240

241

242

243

244

245

246

247

248

249

250

251

252

253

254

255

256

257 Since DISCOVER-Seq can distinguish high-specificity gRNAs from promiscuous gRNAs, we

258 asked if this approach can also distinguish a high-fidelity Cas9 mutant from the less fidelitous wild 259

comparable between $\mathrm{gP}$ and $\mathrm{gP}+\mathrm{G}$ edited cells, but off-target mutation rates differed substantially

(Fig. S4C and Table S2). Thus, DISCOVER-Seq can not only distinguish high-fidelity gRNAs from promiscuous gRNAs, but can also be used to characterize off-target profiles generated by gRNAs with alternative protospacer lengths and modifications.

\section{Cas9 cutting dynamics at on- and off-target sites}

The kinetics of Cas9 cleavage and binding are well-studied in vitro and the amount of mismatches of a protospacer to an on- or off-target site can dramatically affect an RNP's on- and off-rate (36). However, relatively little is known about dynamics of on- and off-target cleavage by Cas9 in a cellular context. Because DISCOVER-Seq measures DSBs rather than finished repair outcomes (indels), we used it to ask if off-target sites are cut simultaneously with the on-target site. We performed a DISCOVER-Seq time-course experiment in B16-F10 cells edited with Cas9 and the promiscuous $\mathrm{gP}+\mathrm{G}$ guide, analyzing samples $8 \mathrm{~h}, 12 \mathrm{~h}, 24 \mathrm{~h}$ and $36 \mathrm{~h}$ post nucleofection (Fig. S4D).

We observed that DSBs at the on-target site were most prominent at the $12 \mathrm{~h}$ time point, supporting our observations from the ChIP-qPCR time course in K562 cells (Fig. 1D and Fig. S1). We found that off-target cleavage dynamics were generally comparable to those at the on-target site and that earlier time points allow a more sensitive detection of off-targets (Fig. S4D).

\section{DISCOVER-Seq characterizes off-target profiles of Cas9 variants}

Several high-fidelity Cas9 variants have been developed that reduce off-target editing (37-39).

type (WT) Cas9. We edited K562 cells with RNPs targeting the previously mentioned $H B B$ locus 
that has two known off-target sites. We formed these RNPs using WT Cas9 and the HiFi Cas9 R691A mutant (38) and compared the DISCOVER-Seq profiles of each experiment. Cells edited with WT Cas9 showed prominent MRE11 peaks at the on-target site in $H B B$ and off-target site OT1, with a smaller peak at the less frequently edited OT2 site. Cells edited with HiFi Cas9 showed comparable MRE11 binding at $H B B$, but a dramatically decreased binding of MRE11 to OT1 and undetectable binding to OT2 (Fig. 4D). Amplicon-NGS verified that WT Cas9 produces indels at $H B B, O T 1$, and $O T 2$, while HiFi-Cas9 primarily edits $H B B$ with greatly reduced indels at $O T 1$ and none at OT2 (Fig. 4E and Fig. S5A and Table S1). To investigate a larger off-target panel we also tested HiFi-Cas9 and DISCOVER-Seq with the promiscuous VEGFA site 2 gRNA in K562 cells. Again, DISCOVER-Seq detected fewer off-targets with the HiFi Cas9 and this was validated with amplicon-NGS (Fig. 4F and Fig. S5B-C and Table S1). Surprisingly, DISCOVER-Seq identified

271 an additional off-target with the HiFi Cas9 that was not found previously by GUIDE-Seq and is

272 not observed with wild-type Cas9 (8) (Fig. 4F). This new off-target was validated by amplicon-

273 NGS and showed $0.75 \%$ indels above background. These data illustrate that high-fidelity Cas9

274 variants can have unanticipated off-target profiles that can be found using DISCOVER-Seq.

\section{Off-target detection in patient-derived stem cells using DISCOVER-Seq}

276 We have so far shown that DISCOVER-Seq works robustly in immortalized mammalian cells.

277 Stem cells, such as induced pluripotent stem cells (iPSCs), are much more sensitive and difficult

278 to culture and transfect, and so are not amenable to methods such as GUIDE-Seq. As DISCOVER-

279 Seq requires nothing but the editing reagents to be introduced into cells, off-target detection should 280 be possible even in patient-derived iPSCs. We derived iPSCs from a patient suffering from 281 Charcot-Marie-Tooth disease (CMT) - one of the most common inherited neurological disorders. 
282 The patient carries a heterozygous missense mutation (P182L) in heat-shock protein B1 (HSPB1)

283 resulting in a dominant negative neuropathic phenotype with a loss of motor neuron function (40).

284 Mice heterozygous for HSPB1 show no disease phenotype, so an attractive therapeutic strategy is

285 to knock out the disease allele without affecting the intact copy of $\operatorname{HSPB1},(41,42)$. For this

286 strategy to move into the clinic, it is important to characterize potential missense-targeting editing

287 reagents in detail, ideally in a patient-specific manner.

288 We edited iPSCs from the CMT patient with HiFi Cas9-RNPs targeting either the HSPB1 wild289 type (WT) or mutant (Mut) allele and subsequently performed DISCOVER-Seq on both sets of 290 edited cells to identify patient-specific and allele-specific off-targets for each gRNA (Fig. 5A-B).

291 For the WT gRNA, we detected the on-target site HSPBI and one major off-target in a non-coding 292 region that contains an exact duplication of HSPB1 exon 3. Both targets were validated by 293 amplicon-NGS after 4 days (Fig. 5C, top, and Table S3). DISCOVER-Seq on patient iPSCs edited 294 with the Mut gRNA exclusively identified the on-target site HSPB1, but not the noncoding off295 target site that contains a protospacer mutation relative to the Mut gRNA (Fig 5C, bottom, and 296 Table S3). These data suggest that the Mut gRNA is highly specific for the disease allele.

297 Because indels introduced to the HSPB1 locus cover the heterozygous patient mutation, simple 298 amplicon-NGS cannot distinguish editing of the WT or Mut allele. Hence, the indel rates reported 299 in Fig. 5C are a summary of editing events on both alleles. We therefore used allele drop-out rates 300 to investigate whether editing was allele specific by examining reads that still contained the intact 301 WT or Mut allele in each of the edited samples. In a control sample of heterozygous patient iPSCs 302 that was edited with a non-targeting gRNA, intact WT and Mut reads accounted for approximately 
$50 \%$ of total aligned reads. By subtracting the intact WT or Mut alleles in the edited samples from the observed 50\% we determined editing frequencies on each allele (Fig. 5D).

We found that cells edited with the WT gRNA showed a substantial reduction of both WT and Mut alleles, suggesting that both alleles were edited to some extent and that the WT gRNA cannot distinguish the two alleles. In contrast, cells edited with the Mut gRNA showed mainly drop-out of Mut alleles but left most WT alleles intact. We then re-purposed DISCOVER-Seq to give us more detailed information about allele-specificity of the WT and Mut gRNAs by examining the sequence of the MRE11 ChIP-Seq reads at the target site. We found that in heterozygous iPSCs

311 edited with the WT gRNA, the DNA bound by MRE11 contained WT sequence but also $30 \%$ of 312 the $\mathrm{G}>\mathrm{A}$ mutation. By contrast, in heterozygous iPSCs edited with the Mut gRNA, 92\% of DNA 313 bound by MRE11 contained the G>A mutation. (Fig. 5E). Overall, these data indicate that the 314 patient-specific $H S P B 1$ gRNA described here is specific for the mutant allele while sparing the

315 WT allele, and that DISCOVER-Seq can be used to investigate both genomic and allelic off-target 316 sites in patient-specific genomes in order to characterize editing reagents for personalized therapy.

\section{DISCOVER-Seq measures CRISPR specificity in vivo during viral-mediated editing of mice}

318 Characterizing CRISPR-Cas off-targets after in vivo gene editing presents a major challenge but 319 is an important milestone for clinical translation of therapeutic editing. Current approaches to test 320 in vivo gRNA specificity rely on in vitro treatment of isolated genomic DNA followed by 321 exhaustive amplicon-NGS testing of hundreds to thousands of potential off-targets (11). While this 322 approach can distinguish promiscuous gRNAs from fidelitous gRNAs, it involves a great deal of 323 experimental effort with many false positives. 
324 We asked if DISCOVER-Seq can be applied to detect off-targets in mouse tissues after in vivo 325 viral-mediated gene editing. We first used Western blotting to find that Mre11 expression is 326 variable but broad across multiple mouse tissues and is indeed more widely expressed than other 327 repair proteins such as Fancd2 (Fig. S6A).

328 In vivo editing outcomes for the $\mathrm{gP}+\mathrm{G}$ promiscuous guide targeting $P$ csk 9 have been characterized 329 in detail by 'verification of in vivo off-targets' (VIVO), and so this gRNA represents a good test 330 bed for in vivo DISCOVER-Seq (11). We used adenoviral infection to deliver gP and WT Cas9 to 331 mice, plus a negative control cohort encoding GFP and Cas9 (Fig. 6A). To note, a 5 ' G is added 332 upon $\mathrm{U} 6$ promoter-driven transcription of $\mathrm{gP}$ that results in $\mathrm{gP}$ expression in vivo as $\mathrm{gP}+\mathrm{G}$, 333 therefore we denote it here as $\mathrm{gP}+\mathrm{G}$. Two mice were sacrificed at each $24 \mathrm{~h}, 36 \mathrm{~h}$ and $48 \mathrm{~h}$ timepoint 334 post viral infection and DISCOVER-Seq was performed on the target liver tissue as well as lung 335 tissue that is not targeted by the adenovirus (11). As $48 \mathrm{~h}$ post infection is too early to reliably 336 detect indels in vivo (Fig. S6B), we used edited livers from three mice that were sacrificed 4 days 337 after infection to determine indel frequencies (Fig. 6A).

338 Using DISCOVER-Seq, we found that cleavage at the on-target Pcsk 9 site in liver was strongest 33924 hours post infection and then declined over time (Fig. 6B and Fig. S6C). Amplicon-NGS 340 showed between 49.3 and $64.4 \%$ indels for the on-target Pcsk9. DISCOVER-Seq confirmed tissue341 specificity of the adenoviral-mediated editing, measuring no Pcsk9 cleavage in lung tissue, even 342 though Mre11 is well-expressed in the lung (Fig. 6B and Fig. S6A). We confirmed by amplicon343 NGS that no indels were present in the lung tissue $(0.042 \%)$. Hence, DISCOVER-Seq is capable 344 of establishing tissue specificity during in vivo viral genome editing. 
345 To account for potential differences in editing and ChIP-Seq timing between animals, we

346 bioinformatically pooled the DISCOVER-Seq reads from the edited mice and analyzed the pooled

347 reads using BLENDER. To normalize for higher coverage with multiple samples, we adjusted our

348 filtering scheme accordingly to limit false positives (Fig. S3). DISCOVER-Seq identified 36 off-

349 target sites in the liver samples and we followed up on 27 of them (Fig. 6C and Table S4).

350 Amplicon-NGS and Sanger sequencing confirmed that all 27 off-targets that we tested were edited,

351 with indel frequencies ranging from $0.9 \%$ to $78.1 \%$. This illustrates that DISCOVER-Seq

352 exclusively identifies bona-fide off-targets (Fig. 6C and Table S4). Low frequency off-targets

$353(<0.8 \%$ indels $)$ that were determined as significant by VIVO could not be detected by DISCOVER-

354 Seq. However, 17 of the bona-fide off-target sites found by DISCOVER-Seq and validated by

355 amplicon NGS (indel frequencies ranging from 0.95 to $51 \%$ ) were not previously characterized by

356 VIVO. While CIRCLE-Seq did identify these sites, their CIRCLE-Seq score was relatively low

357 and they could have been missed among the noise of the $>3000$ sites identified by CIRCLE-Seq.

358 By contrast, DISCOVER-Seq unbiased detection found only true off-targets during viral-mediated

359 in vivo gene editing in a single experimental workflow.

\section{Discussion}

361 DISCOVER-Seq is a universal approach for the unbiased detection of genome editing off-targets

362 that is applicable in cell lines, primary cells, and tissues edited with multiple delivery systems in

363 human and mouse. We find that ChIP-Seq of DNA repair proteins can spatially and temporally

364 characterize Cas-induced DNA damage on a molecular level. In particular, MRE11 ChIP-Seq 365 provides valuable insights into SpCas9 and AsCas12a cleavage patterns and dynamics. We used 366 these data to characterize Cas nuclease activity in living cells. Our data reveal that the PAM side 
367 of a Cas9-induced DSB is more protected from resection. Since Cas9 stays tightly bound to DNA

368 in vitro $(29,30,43,44)$ and in vivo $(19,20)$ even after a DSB has occurred, we favor a model in

369 which the Cas9 protein itself protects the DNA ends on the PAM side from being accessed by

370 other nucleases which could influence repair of the break. Cas9 can itself make non-blunt cuts on

371 the PAM-distal side, but the degree of resection we observe in the MRE11 ChIP-Seq is far greater

372 than the few bases of imprecision observed for in vitro Cas9 cutting (31). Interestingly, we found

373 no asymmetry after AsCas12a editing, although more data from multiple different Cas12a gRNAs

374 will be needed to support this hypothesis.

375 DISCOVER-Seq gives insight into the dynamics of Cas9 cutting at on- and off-target sites after

376 RNP editing in cells and after adenoviral editing in mice. This is in contrast to previous cellular

377 kinetics experiments (45), which mainly examined the products of repair. We found that DSBs

378 and MRE11 binding occur very early on after RNP editing (4-12h post nucleofection) and as early

379 as $24 \mathrm{~h}$ after viral infection in tissues. In vitro Cas9 resides significantly longer at the on-target site

380 than at off-target sites, resulting in more efficient cleavage $(19,20,36)$. However, our ability to

381 study cleavage products in cells suggest that in this context the on-target and off-target cutting

382 dynamics are not significantly different. Several factors could explain this discrepancy. Timing

383 may be specific to particular off-target loci, concentrations of Cas9 used in different experiments

384 may influence enzyme availability for rarer off-targets, or the discrepancy in timescales between

385 in vitro experiments (minutes) and in vivo experiments (hours to days) may push reactions toward

386 equilibrium. So far, our data suggest that Cas9 does not temporally distinguish between on and

387 off-targets in cells. 
In vitro assays such as CIRCLE-Seq (6) and SITE-Seq (7) strip the DNA of all bound proteins before nuclease digestion, resulting in efficient cleavage of all potential sites by the Cas9 enzyme. These assays can detect potential off-targets with incredible sensitivity, but cannot provide information about how in vitro cleaved sites translate into bona-fide off-targets in cellular models. complexes all influence Cas9 cutting efficiency and thus off-target mutagenesis in vivo (14, 30, 40). It is currently difficult to predict repair outcomes from in vitro data. Performing in vitro assays and then validating all potential cleavage sites by amplicon-NGS in vivo is subject to high false positive rates and can be extremely laborious depending on the number of potential sites, particularly when a single CIRCLE-Seq experiment can return as many as 3000 putative off-targets (11). DISCOVER-Seq instead directly provides data on true Cas9 cleavage events, as it measures off-targets within the edited cell in its original chromatin state. We have also found that DISCOVER-Seq robustly identifies true in vivo off-targets that are missed by CIRCLE-Seq or GUIDE-Seq due to poor ranking among hundreds to thousands of false positives. DISCOVER-

402 Seq enables one to determine bona-fide off-targets in a one-step procedure, which reduces the number of target sites to be validated by amplicon-NGS by orders of magnitude. The in vivo sensitivity of DISCOVER-Seq is not as high as in vitro CIRCLE-Seq, as off-targets below $0.8 \%$

405 indels were missed. However, this could be due to the sensitivity of ChIP-Seq in tissues and might

406 be potentially improved by using more sensitive ChIP methods such as CUT\&RUN (47) or by 407 increasing the overall read depth of the Illumina Sequencing. MRE11 ChIP-Seq yields provocative data regarding the error-prone nature of Cas9 break repair.

409 While indels formed by Cas9 involve error-prone repair, it is currently unclear to what extent these 410 outcomes are the end-product of multiple rounds of error-free repair after genomic cleavage (16, 
$41145,48)$. Some gRNAs that exhibit low indel efficiency may actually cut efficiently but breaks

412 could be repaired perfectly. This would be a complicating factor given that such sites would be

413 overlooked when considering possible genomic translocations (49). DISCOVER-Seq detects off-

414 target events prior to repair and is thus independent of the repair outcome. In the future,

415 DISCOVER-Seq could also be used to find sites of Cas9 action that could be hot spots for

416 translocations, rearrangements and larger deletions that do not result in small indels at the cut site.

417 While DISCOVER-Seq signal is generally correlated with indel formation (Fig. S4A), there are

418 notable exceptions. We found several target sites that showed disproportionately high MRE11-

419 bound DNA fragments with low indels. For example the D6Ertd527e off-target in gP-edited B16-

420 F10 cells exhibited a larger MRE11 peak than the on-target Pcsk9 site, the off-target had only

$421 \sim 25 \%$ indels compared to $\sim 66 \%$ indels at the on-target. More work remains to be done to determine

422 if MRE11 binding can truly find sites that are perfectly repaired at high frequency, or if there are

423 unknown limits to the predictive power between MRE11 binding and indel formation.

424 In summary, DISCOVER-Seq is broadly applicable to detect off-targets from all tested Cas

425 variants in multiple species. It should also be applicable to other classes of nucleases such as

426 Transcription activator-like effector nucleases (TALENs) and Zinc finger nucleases (ZFNs),

427 though we have not tested these explicitly. The biological function of the MRN complex is highly

428 conserved among species and even across domains and one could imagine applying a similar off-

429 target detection strategy in plants or other organisms. Moreover, DISCOVER-Seq's ability to

430 visualize Cas enzyme activity in a cellular context has numerous applications beyond off-target

431 discovery, including understanding CRISPR-Cas cutting patterns and dynamics in vivo and

432 shedding light onto DSBs and repair outcomes in different cell types and tissues. DISCOVER-Seq

433 can even be used to characterize clinical editing reagents and, in particular, to identify off-targets 
434 from personal genotypes uncovering how normal genetic variation affects editing accuracy. We

435 also anticipate that it could potentially be used for real-time off-target discovery in patient biopsy

436 samples after in vivo editing in the clinic.

\section{Acknowledgements}

438 J.E.C., B.W., C.D.R., S.K.W., C.D.Y. and K.R.K. are supported by the Li Ka Shing Foundation

439 and Heritage Medical Research Institute. J.E.C. is supported by the National Heart, Lung, and

440 Blood Institute of the NIH under DP2-HL-141006. J.T.V. is supported by CIRM TRAN1-09292.

441 B.W. is supported by a Sir Keith Murdoch Fellowship from the American Australian Association.

442 This work used the Vincent J. Coates Genomics Sequencing Laboratory at UC Berkeley, supported

443 by NIH S10 OD018174 Instrumentation Grant. We would like to thank Marie Johansson for 444 technical support on intravenous tail vein injections and the Gladstone Stem Cell Core for their 445 services.

\section{Conflicts of interest}

447 P.A., M.Morlock., M.J.P., and M.Maresca are employees and shareholders of AstraZeneca.

\section{Contributions}

449 B.W., S.K.W., C.D.R. and J.E.C. conceived and designed the study and experiments. B.W. 450 performed ChIP-Seq, PCRs for amplicon-NGS and Western Blots in cell lines. S.K.W. analyzed

451 ChIP-Seq and amplicon-NGS data and developed BLENDER software. C.D.Y. performed ChIP452 qPCR time course experiments. K.R.K. prepared ChIP-Seq libraries. J.V.T. prepared amplicon- 
453 NGS libraries. P.A., M.J.P. and M. Morlock executed intravenous tail vein injections, animal

454 terminations and organ collection and performed Western Blots in mouse tissues. L.M.J. and

455 H.L.W. planned and performed iPSC editing experiments. M. Maresca and B.R.C. supervised

456 experiments and provided expertise. J.E.C. supervised the study. B.W., S.K.W. and J.E.C wrote

457 the manuscript with input from all authors. 


\section{Main figures}

\section{Figure 1}

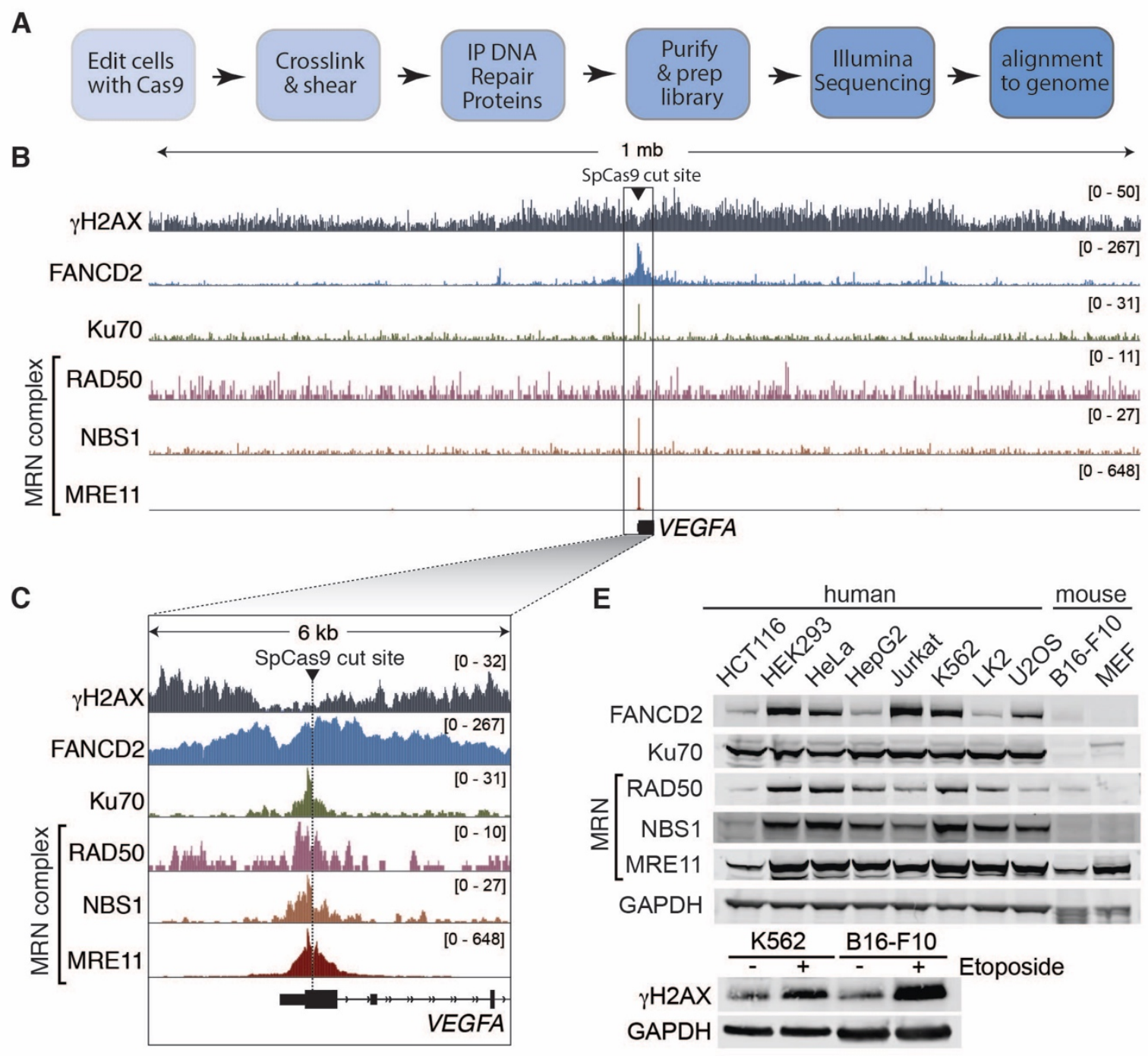

D

On-target $H B B$

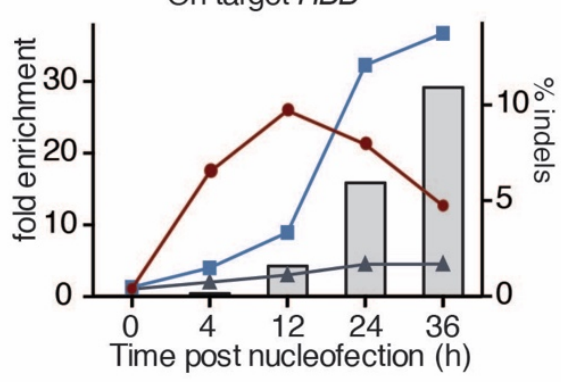

Off-target OT1

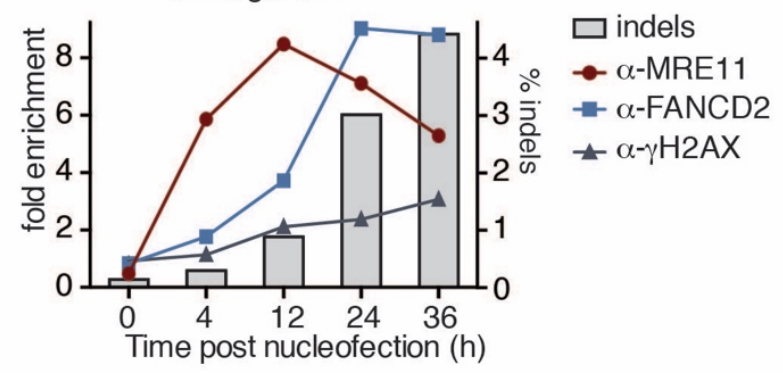

Figure 1: DNA repair proteins assemble at a Cas-induced DSB. (A) Schematic of workflow 
463 FANCD2, Ku70, NBS1, RAD50, and MRE11 accumulate at SpCas9 cut sites in cells. DNA repair 464 proteins were localized and quantified by ChIP-Seq using the indicated repair proteins in K562 465 cells and plotting ChIP-Seq reads over a $1 \mathrm{Mb}$ window spanning the VEGFA cut site. Inset numbers 466 define the height of the y-axis in reads. (C) Height and width of ChIP-Seq signals vary widely for 467 DNA repair proteins. Data presented as described in Fig. 1B, except the windowed region is $6 \mathrm{~kb}$. 468 The SpCas9 target site is marked with a dotted line. (D) DNA repair proteins are present at Cas9469 induced breaks with varying kinetics. ChIP-qPCR experiment for MRE11, FANCD2 and $\gamma \mathrm{H} 2 \mathrm{AX}$ 470 in K562 cells edited at $\mathrm{t}=0$ with an RNP targeting the $H B B$ gene. Cells were harvested for ChIP at 471 indicated time points and DNA extracted. The fold enrichment is the relative binding of each factor 472 at the on-target site $(H B B)$ or a known off-target site $(O T 1)$ normalized to a negative control region $473(V E G F A)$. The bar graph depicts the amount of indels as determined by amplicon-NGS. Shown is 474 one replicate, a second replicate is shown in Fig. S1. (E) Protein levels of DNA repair proteins 475 across a variety of human cell lines from different origins and cross-reactivity of the tested 476 antibody with two murine cell lines. Whole cell lysates from untreated cells were analyzed for 477 FANCD2, Ku70, MRE11, NBS1 and RAD50 abundance by Western Blot. GAPDH abundance is 478 used as loading control. To induce $\gamma \mathrm{H} 2 \mathrm{AX}$ phosphorylation, cells were treated with Etoposide for $47916 \mathrm{~h}$ before protein extraction. 


\section{Figure 2}

A

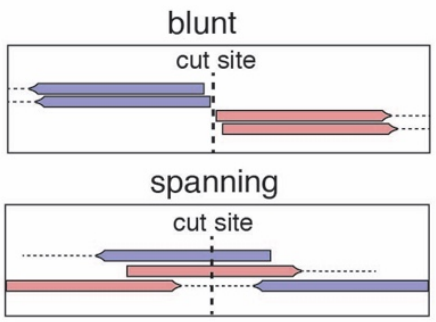

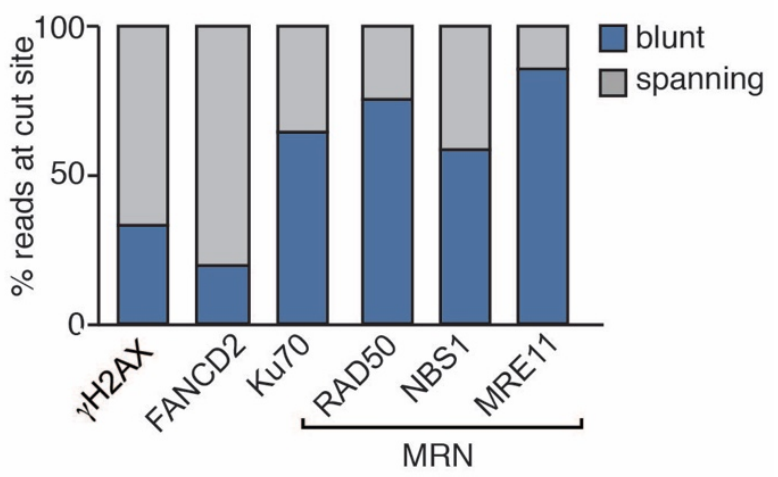

B
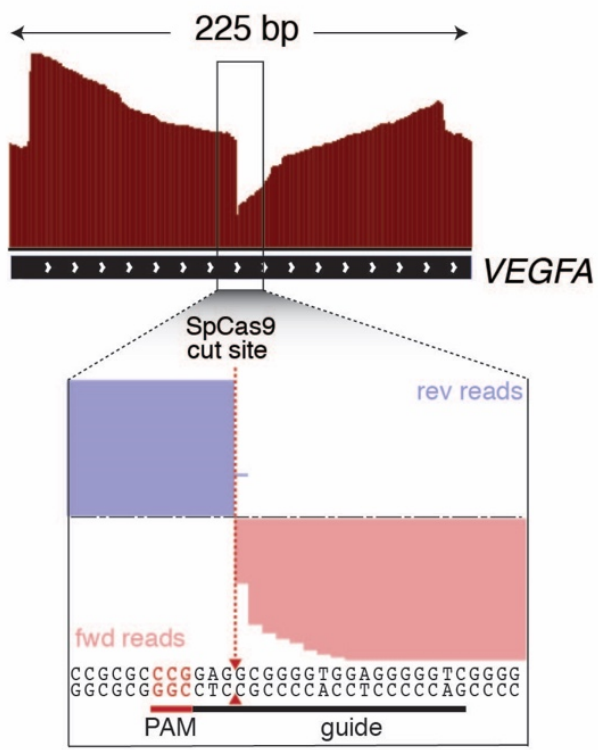

D

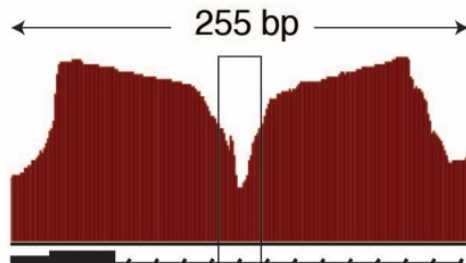

$255 \mathrm{bp}$

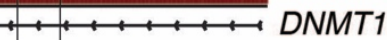

\section{AsCas12a} cut sites

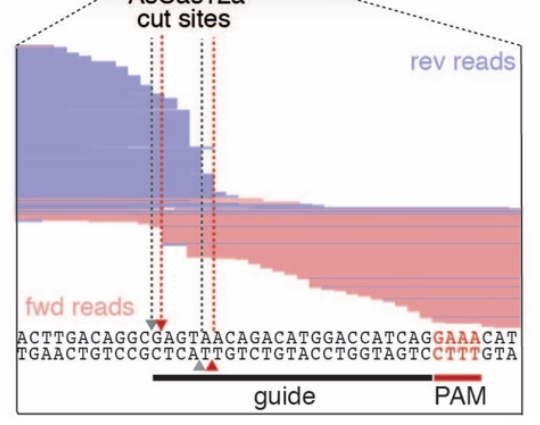

C
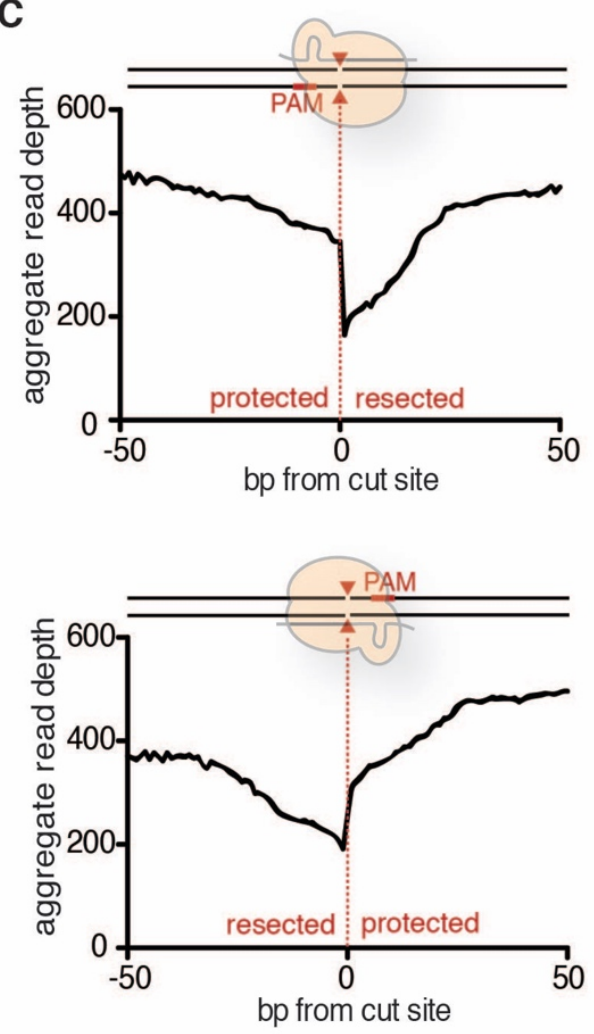


\section{Figure 2: ChIP-Seq molecularly characterizes Cas-induced breaks at single-base resolution.}

483 (A) Schematic of different types of reads found at the cut site (left) and composition of aligned 484 ChIP-Seq reads at the on-target cut site $(V E G F A)$ for each DNA repair factor (right). Shown is the 485 fraction of read pairs that end or start within a $10 \mathrm{bp}$ window around the cut site (blunt) and the 486 fraction of read pairs that are spanning the cut site (spanning). (B) MRE11 peaks are asymmetric. 487 MRE11 ChIP-Seq signal is presented as described in Fig. 1B over a 225 bp interval spanning the 488 cut site. The windowed region (black box) shows aligned individual Illumina Sequencing reads in 489 forward (pink) or reverse (purple) direction. MRE11 peaks have a distinct shape due to reads 490 ending or starting in the Cas9-induced cut site. Peaks are markedly asymmetric, since all reads 491 perfectly abut one side of the break but many reads are resected from the other side (see Fig. S2A 492 for more detail). (C) Accumulated read depth of MRE11 ChIP-Seq peaks in K562 cells edited with 493 SpCas9-RNPs targeting VEGFA. Reads are accumulated over multiple on- and off-target sites, 494 binned into whether the gRNA binds the sense strand (eight sites) or the gRNA binds the antisense 495 strand (eight sites). Reads are blunt against the PAM-containing end of the DNA, but are both 496 blunt and resected on the non-PAM end. (D) MRE11 ChIP-Seq in K562 cells edited with an 497 AsCas12a-RNP targeting DNMT1. Enlarged is a region around the AsCas12a cut site showing the 498 composition of aligned reads. The read distribution matches the activity of AsCas12a to produce 499 a 5' overhang. A predicted 4 nucleotide AsCas12a cut site is marked in grey and a 5 nucleotide 500 alternative cut site is shown in red. 


\section{Figure 3}

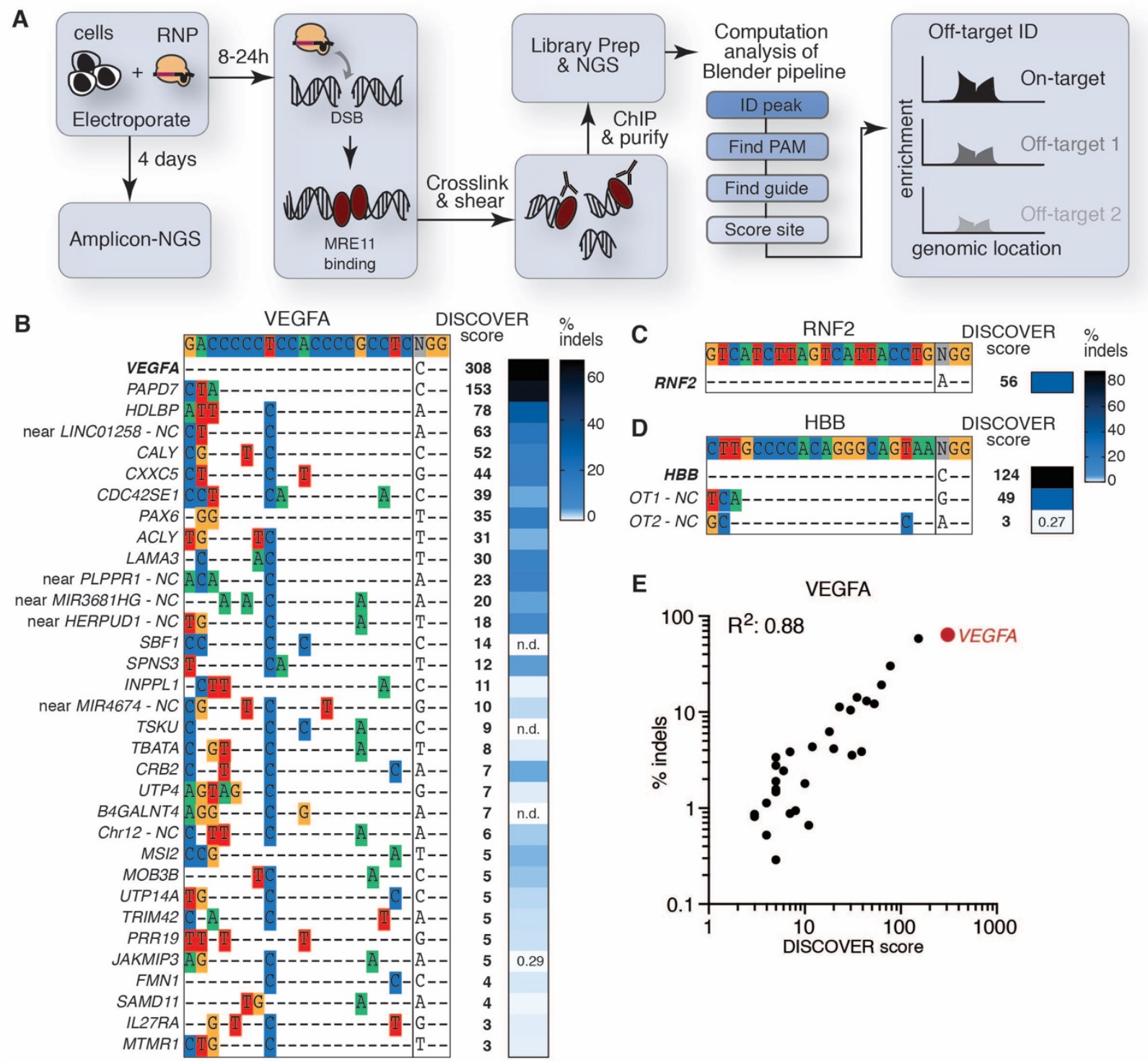

Figure 3: Unbiased off-target discovery in human cells using DISCOVER-Seq. (A) Schematic

505 showing the general workflow of DISCOVER-Seq. The first part of DISCOVER-Seq consists of genome editing of cells followed by MRE11 ChIP-Seq at a relatively short time point to capture

507 Cas-induced DSBs. The data is then input to a custom bioinformatics pipeline (BLENDER) to

508 determine the genomic location of off-target sites (see Fig. S3 for more detail). (B) Sequences of 509 off-targets identified with DISCOVER-Seq for VEGFA site 2 in K562 cells. The on-target 510 sequence and site is shown on the top and discovered off-target cleavage sites are beneath. Any 
511 mismatches to the on-target sequence are highlighted in color. DISCOVER scores and indel 512 frequencies compared to unedited cells as determined by amplicon-NGS are shown to the right for 513 each site.. DISCOVER-Seq finds bona fide off-target sites verifiable with amplicon-NGS and does 514 not report false positive sites. NC: non-coding. n.d.: not determined due to PCR difficulties. (C) 515 and (D) Sequences, DISCOVER scores and indel frequencies of off-targets identified with

516 DISCOVER-Seq for RNF2 and HBB targeting gRNAs in K562 cells. (E) DISCOVER scores and 517 indel frequencies are highly correlated. Relationship between VEGFA off-target indel frequencies 518 and DISCOVER score for each off-target. VEGFA on-target site is highlighted in red. 


\section{Figure 4}
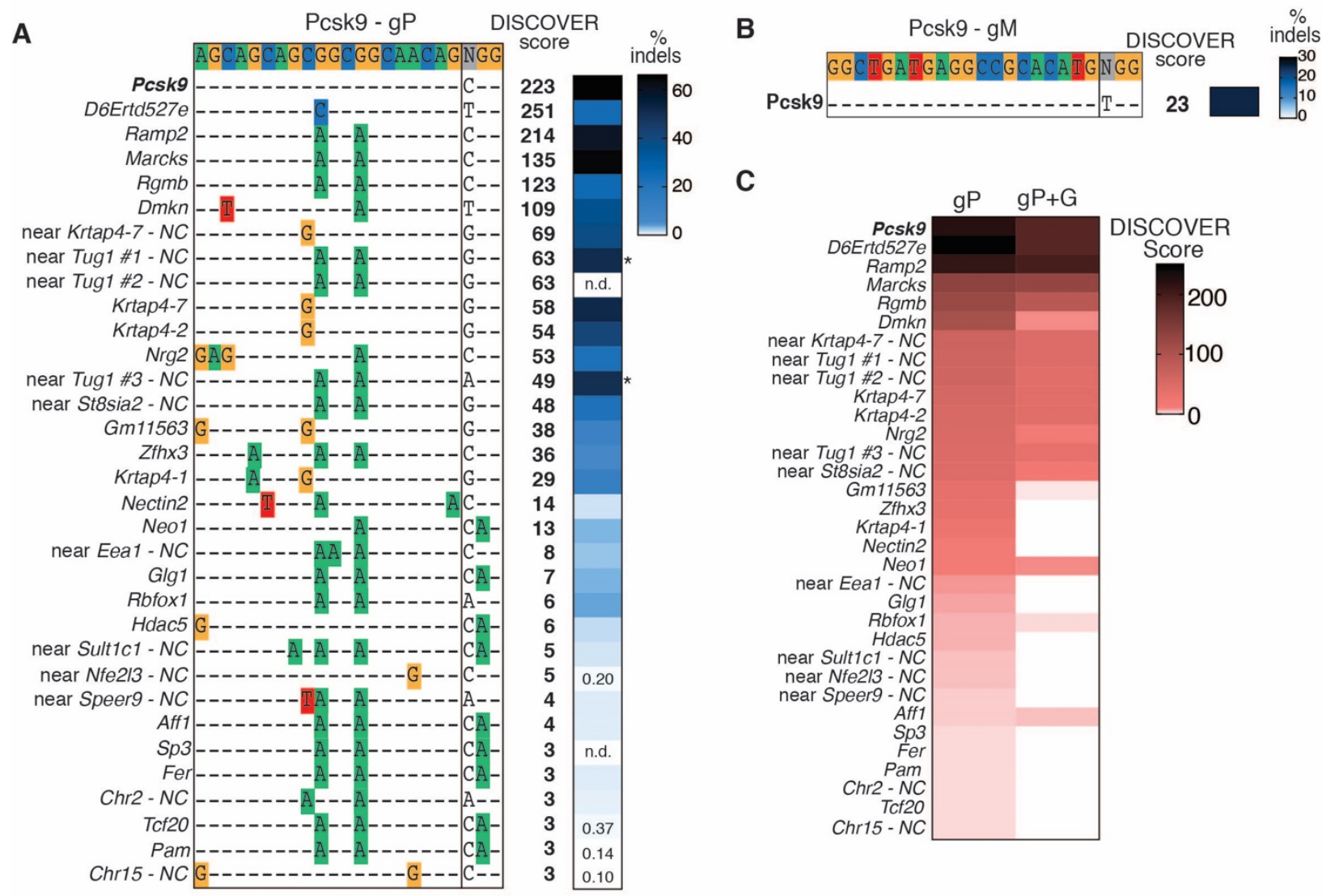

C

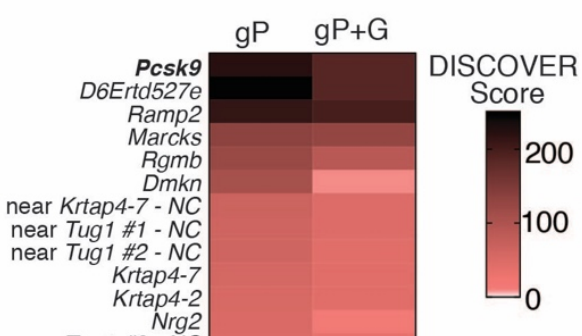

near Tug1 \#3 - NC

near St8sia2 - NC

Gm11563

Gm11563

Zthx3

Krtap4-1
Nectin2

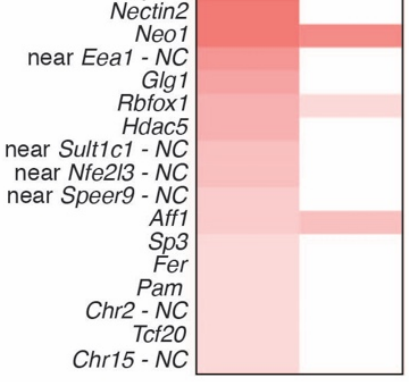

200

00

Chr15 - NC

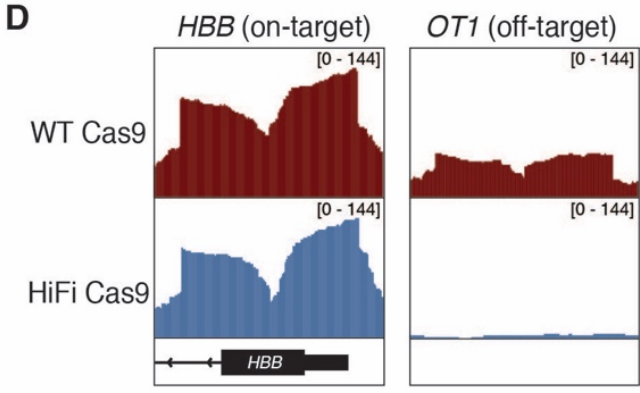

E

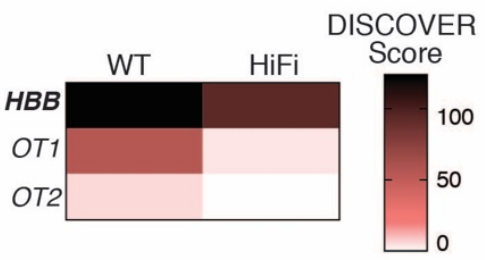


521 Figure 4: DISCOVER-Seq is broadly applicable. (A) Sequences of off-targets for the Pcsk9 gP

522 gRNA identified with DISCOVER-Seq in murine B16-F10 cells. The on-target sequence and site

523 is shown on the top and discovered off-target cleavage sites are beneath. Any mismatches to the

524 on-target sequence are highlighted in color. DISCOVER scores and indel frequencies compared

525 to unedited cells as determined by amplicon-NGS are shown to the right for each site. Indel

526 frequencies marked with an asterisk were not reliable by amplicon-NGS and were instead

527 determined by Sanger sequencing and ICE analysis. NC: non-coding; n.d.: not determined. (B)

528 Sequence, DISCOVER score, and indel frequency of the single target for the Pcsk9 gM gRNA

529 identified with DISCOVER-Seq in murine B16-F10 cells. (C) DISCOVER scores for off-targets

530 of the $P \operatorname{csk} 9 \mathrm{gP}$ gRNA and a $P \operatorname{csk} 9 \mathrm{gP}$ gRNA with an additional 5' $\mathrm{G}$ added to the protospacer

$531(\mathrm{gP}+\mathrm{G})$ in B16-F10 cells. The addition of the 5, $\mathrm{G}$, commonly added for efficient in vitro

532 transcription and plasmid or viral transcription, has a large effect on the number of off-targets. (D)

533 MRE11 ChIP-Seq peaks for K562 cells edited with WT Cas9 or HiFi Cas9 and HBB gRNA at the

534 on-target $(H B B)$ and a known off-target site (OT1). (E) DISCOVER scores for off-targets from

535 K562 cells edited with WT Cas9- or HiFi Cas9-RNPs targeting HBB. (F) DISCOVER scores for

536 off-targets from K562 cells edited with WT Cas9- or HiFi Cas9-RNPs targeting VEGFA (VEGFA

537 site 2). A black diamond indicates an off-target that was specific for the sample edited with HiFi

538 Cas9 and has not been previously described.

539 


\section{Figure 5}

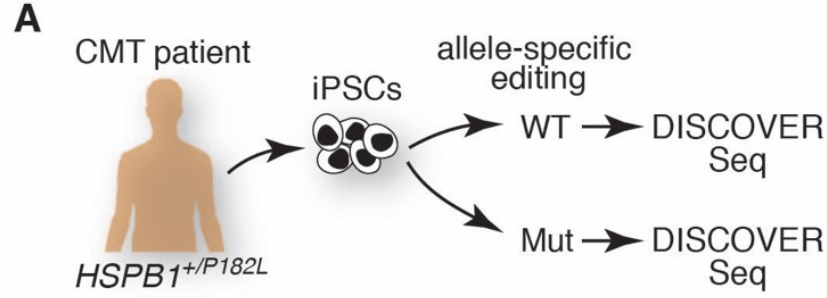

C

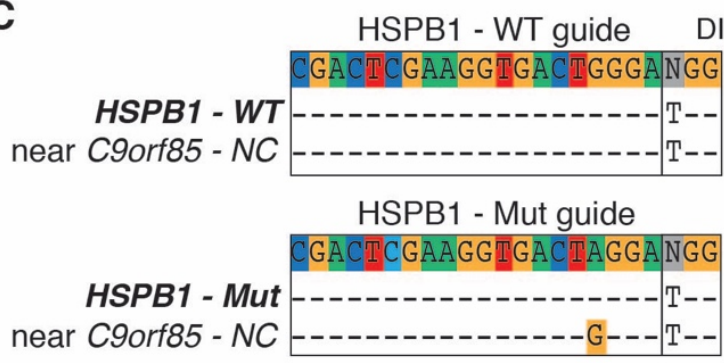
8 9
B
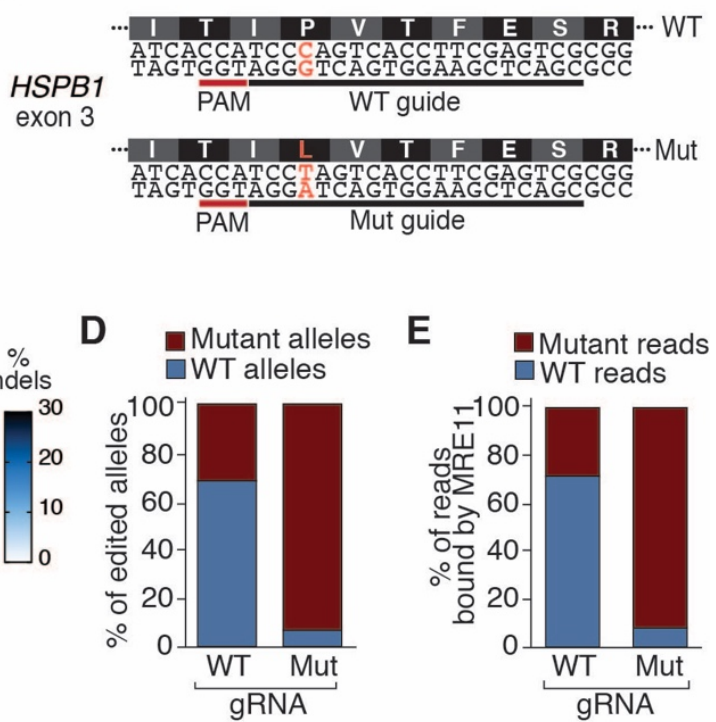

Figure 5: DISCOVER-Seq off-target discovery in patient-derived pluripotent stem cells. (A)

Schematic showing experimental workflow. iPSCs were derived from a Charcot-Marie-Tooth (CMT) patient heterozygous for the disease mutation P182L in the HSPB1 gene. iPSCs were edited with HiFi Cas9 and two different gRNAs targeting either the WT or the mutant allele as shown in (B). Off-targets from each gRNA were analyzed using DISCOVER-Seq. (B) Schematic of alleles in patient iPSCs and targeting gRNAs. (C) Sequences, DISCOVER scores, and indel frequencies of off-targets for HSPBI WT and HSPBI mutant (Mut) gRNAs in heterozygous patient iPSCs. The WT gRNA edits both $H S P B 1$ as well as a perfectly matching site in a noncoding region, while the Mut gRNA only edits $H S P B 1$. (D) Allele-specificity of WT and Mut gRNAs was determined by amplicon-NGS. Shown is the fraction of WT and mutant alleles that contain indels after editing with each respective gRNA. Editing with WT gRNA resulted in substantial editing of both WT and mutant alleles while the Mut gRNA almost exclusively targeted the mutant allele. (E) Allelespecific editing efficiency correlates with MRE11 recruitment to HSPB1 cut sites. Shown is the fraction of MRE11 ChIP-Seq reads at the HSPB 1 site that contains either WT or the Mut sequence. 


\section{Figure 6}

A

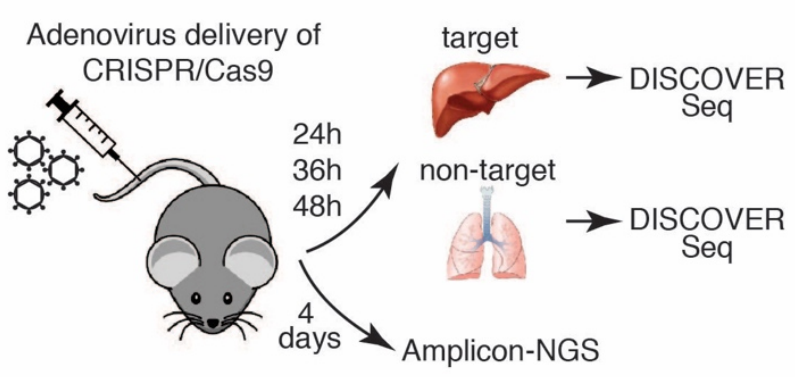

B

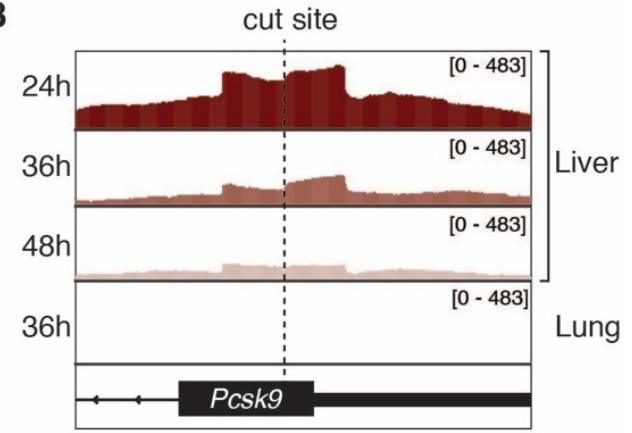

C

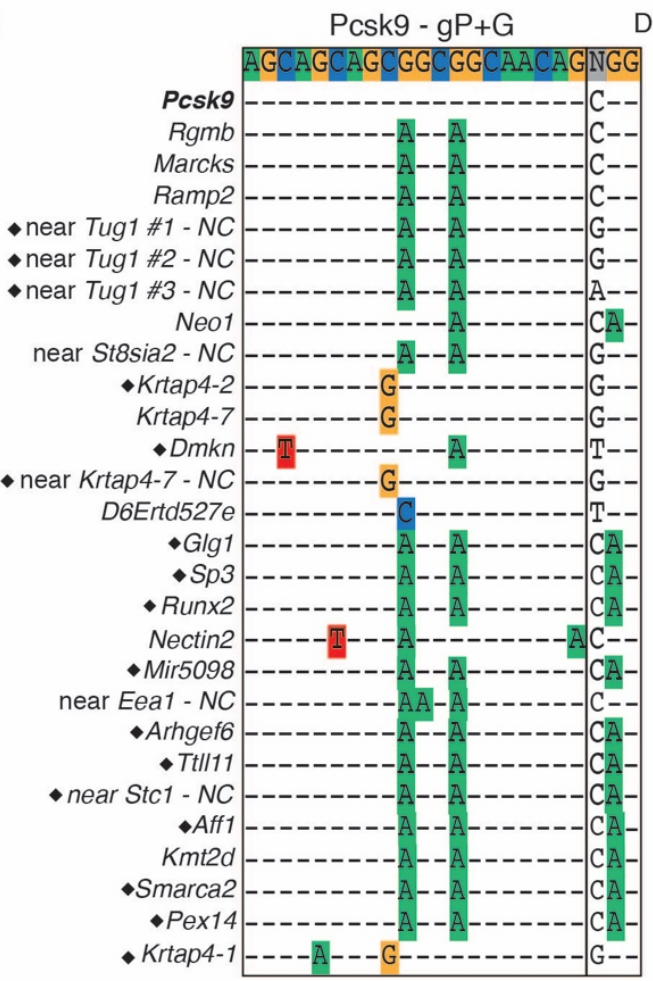

DISCOVER

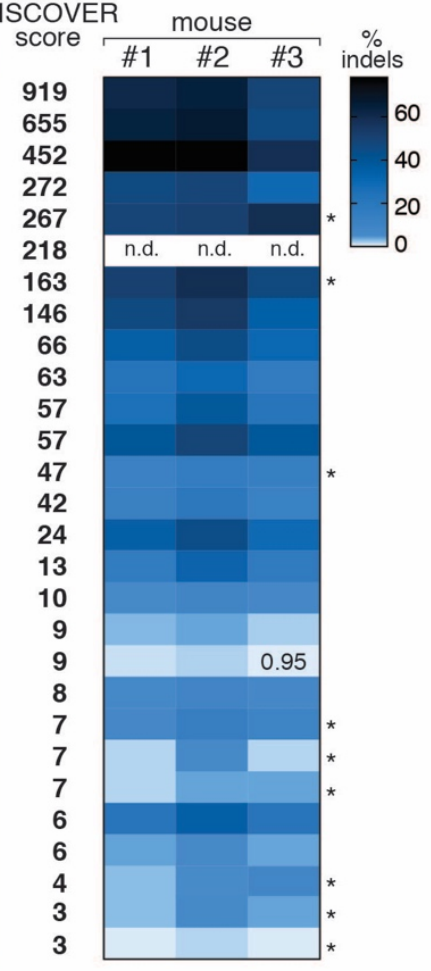

Fig. 6: DISCOVER-Seq identifies genome editing specificity at the genetic and tissue level in vivo. (A) Schematic illustrating the DISCOVER-Seq workflow for in vivo gene editing. Multiple mice were injected with hepatotrophic adenovirus encoding either Cas 9 and a targeting gRNA

564 (Pcsk9 $\mathrm{gP}+\mathrm{G})$ or a negative control virus encoding Cas9 and GFP. On-target tissues (liver) and 565 off-target tissues (lung) were harvested 24, 36 and 48h after injection and DISCOVER-Seq was performed on the tissues. Livers from injected mice after 4 days were used for amplicon-NGS. (B)

567 DISCOVER-Seq accurately distinguishes editing in target and non-target tissues. Mre11 ChIP-

568 Seq signals at the Pcsk9 on-target site in mouse livers at different time points (top) and lung 569 (bottom). No Mre11 binding was detected at the on-target site in the off-target tissue. Shown are 
570 results from three mice at various timepoints, with three additional mice in Fig. S6B. (C)

571 DISCOVER-Seq identifies sites of on- and off-target editing in vivo. Unbiased off-targets

572 identified by DISCOVER-Seq for Pcsk $9 \mathrm{gP}+\mathrm{G}$ in mouse livers. The on-target sequence and site is

573 shown on the top and identified off-target sites are beneath. Mismatches to the on-target sequence

574 are highlighted in color. DISCOVER scores and indel frequencies compared to control mice after

5754 days as determined by amplicon-NGS or Sanger sequencing and ICE analysis (noted with *) are

576 shown to the right for each site. DISCOVER results from pooled sequencing file of all mice are

577 shown. Black diamonds mark sites that were not previously characterized by VIVO (11). NC: non-

578 coding; n.d.: not determined.

579

580

581 


\section{References}

1. W.-J. Dai et al., CRISPR-Cas9 for in vivo Gene Therapy: Promise and Hurdles. Mol. Ther. Nucleic Acids. 5, e349 (2016).

2. S. Bae, J. Park, J.-S. Kim, Cas-OFFinder: a fast and versatile algorithm that searches for potential off-target sites of Cas9 RNA-guided endonucleases. Bioinformatics. 30, 14731475 (2014).

3. S. Abadi, W. X. Yan, D. Amar, I. Mayrose, A machine learning approach for predicting CRISPR-Cas9 cleavage efficiencies and patterns underlying its mechanism of action. PLoS Comput. Biol. 13, e1005807 (2017).

4. J.-P. Concordet, M. Haeussler, CRISPOR: intuitive guide selection for CRISPR/Cas9 genome editing experiments and screens. Nucleic Acids Res. 46, W242-W245 (2018).

5. D. Kim et al., Digenome-seq: genome-wide profiling of CRISPR-Cas9 off-target effects in human cells. Nat. Methods. 12, 237-43, 1 p following 243 (2015).

6. S. Q. Tsai et al., CIRCLE-seq: a highly sensitive in vitro screen for genome-wide CRISPRCas9 nuclease off-targets. Nat. Methods. 14, 607-614 (2017).

7. P. Cameron et al., Mapping the genomic landscape of CRISPR-Cas9 cleavage. Nat. Methods. 14, 600-606 (2017).

8. S. Q. Tsai et al., GUIDE-seq enables genome-wide profiling of off-target cleavage by CRISPR-Cas nucleases. Nat. Biotechnol. 33, 187-197 (2015).

9. W. X. Yan et al., BLISS is a versatile and quantitative method for genome-wide profiling of DNA double-strand breaks. Nat Commun. 8, 15058 (2017).

10. J. Lin, K.-C. Wong, Off-target predictions in CRISPR-Cas9 gene editing using deep learning. Bioinformatics. 34, i656-i663 (2018).

11. P. Akcakaya et al., In vivo CRISPR editing with no detectable genome-wide off-target mutations. Nature. 561, 416-419 (2018).

12. S. Kim, D. Kim, S. W. Cho, J. Kim, J.-S. Kim, Highly efficient RNA-guided genome editing in human cells via delivery of purified Cas9 ribonucleoproteins. Genome Res. 24, 1012-1019 (2014).

13. D. Kim et al., Genome-wide analysis reveals specificities of Cpf1 endonucleases in human cells. Nat. Biotechnol. 34, 863-868 (2016).

14. E. M. Kallimasioti-Pazi et al., Heterochromatin delays CRISPR-Cas9 mutagenesis but does not influence repair outcome. BioRxiv (2018), doi:10.1101/267690.

15. R. M. Yarrington, S. Verma, S. Schwartz, J. K. Trautman, D. Carroll, Nucleosomes inhibit target cleavage by CRISPR-Cas9 in vivo. Proc. Natl. Acad. Sci. USA. 115, 9351-9358 (2018).

16. C. D. Richardson, G. J. Ray, N. L. Bray, J. E. Corn, Non-homologous DNA increases gene disruption efficiency by altering DNA repair outcomes. Nat Commun. 7, 12463 (2016).

17. H. O'Geen, I. M. Henry, M. S. Bhakta, J. F. Meckler, D. J. Segal, A genome-wide analysis of Cas9 binding specificity using ChIP-seq and targeted sequence capture. Nucleic Acids Res. 43, 3389-3404 (2015).

18. X. Wu et al., Genome-wide binding of the CRISPR endonuclease Cas9 in mammalian cells. Nat. Biotechnol. 32, 670-676 (2014).

19. S. C. Knight et al., Dynamics of CRISPR-Cas9 genome interrogation in living cells. Science (80-. ). 350, 823-826 (2015).

20. H. Ma et al., CRISPR-Cas9 nuclear dynamics and target recognition in living cells. J. Cell Biol. 214, 529-537 (2016). 
21. R. Aleksandrov et al., Protein dynamics in complex DNA lesions. Mol. Cell. 69, 10461061.e5 (2018).

22. R. Shroff et al., Distribution and dynamics of chromatin modification induced by a defined DNA double-strand break. Curr. Biol. 14, 1703-1711 (2004).

23. J. S. Iacovoni et al., High-resolution profiling of gammaH2AX around DNA double strand breaks in the mammalian genome. EMBO J. 29, 1446-1457 (2010).

24. R. S. Williams, J. S. Williams, J. A. Tainer, Mre11-Rad50-Nbs1 is a keystone complex connecting DNA repair machinery, double-strand break signaling, and the chromatin template. Biochem Cell Biol. 85, 509-520 (2007).

25. J. Kobayashi, Molecular mechanism of the recruitment of NBS1/hMRE11/hRAD50 complex to DNA double-strand breaks: NBS1 binds to gamma-H2AX through FHA/BRCT domain. J Radiat Res. 45, 473-478 (2004).

26. C. D. Richardson et al., CRISPR-Cas9 genome editing in human cells occurs via the Fanconi anemia pathway. Nat. Genet. 50, 1132-1139 (2018).

27. T. J. Cradick, E. J. Fine, C. J. Antico, G. Bao, CRISPR/Cas9 systems targeting $\beta$-globin and CCR5 genes have substantial off-target activity. Nucleic Acids Res. 41, 9584-9592 (2013).

28. M. A. DeWitt et al., Sci Transl Med, in press, doi:10.1126/scitranslmed.aaf9336.

29. C. D. Richardson, G. J. Ray, M. A. DeWitt, G. L. Curie, J. E. Corn, Enhancing homologydirected genome editing by catalytically active and inactive CRISPR-Cas9 using asymmetric donor DNA. Nat. Biotechnol. 34, 339-344 (2016).

30. R. Clarke et al., Enhanced Bacterial Immunity and Mammalian Genome Editing via RNAPolymerase-Mediated Dislodging of Cas9 from Double-Strand DNA Breaks. Mol. Cell. 71, 42-55.e8 (2018).

31. M. Jinek et al., A programmable dual-RNA-guided DNA endonuclease in adaptive bacterial immunity. Science (80-. ). 337, 816-821 (2012).

32. M. W. Shen et al., Predictable and precise template-free CRISPR editing of pathogenic variants. Nature (2018), doi:10.1038/s41586-018-0686-x.

33. M. van Overbeek et al., DNA Repair Profiling Reveals Nonrandom Outcomes at Cas9Mediated Breaks. Mol. Cell. 63, 633-646 (2016).

34. A. Taheri-Ghahfarokhi et al., Decoding non-random mutational signatures at Cas9 targeted sites. Nucleic Acids Res. 46, 8417-8434 (2018).

35. B. Zetsche et al., Cpf1 is a single RNA-guided endonuclease of a class 2 CRISPR-Cas system. Cell. 163, 759-771 (2015).

36. E. A. Boyle et al., High-throughput biochemical profiling reveals sequence determinants of dCas9 off-target binding and unbinding. Proc. Natl. Acad. Sci. USA. 114, 5461-5466 (2017).

37. B. P. Kleinstiver et al., High-fidelity CRISPR-Cas9 nucleases with no detectable genomewide off-target effects. Nature. 529, 490-495 (2016).

38. C. A. Vakulskas et al., A high-fidelity Cas9 mutant delivered as a ribonucleoprotein complex enables efficient gene editing in human hematopoietic stem and progenitor cells. Nat. Med. 24, 1216-1224 (2018).

39. I. M. Slaymaker et al., Rationally engineered Cas9 nucleases with improved specificity. Science (80-. ). 351, 84-88 (2016).

40. O. V. Evgrafov et al., Mutant small heat-shock protein 27 causes axonal Charcot-MarieTooth disease and distal hereditary motor neuropathy. Nat. Genet. 36, 602-606 (2004). 
674 41. T. Geuens et al., Mutant HSPB1 causes loss of translational repression by binding to PCBP1, an RNA binding protein with a possible role in neurodegenerative disease. Acta Neuropathol. Commun. 5, 5 (2017).

42. L. Huang, J.-N. Min, S. Masters, N. F. Mivechi, D. Moskophidis, Insights into function and regulation of small heat shock protein 25 (HSPB1) in a mouse model with targeted gene disruption. Genesis. 45, 487-501 (2007).

43. S. H. Sternberg, S. Redding, M. Jinek, E. C. Greene, J. A. Doudna, DNA interrogation by the CRISPR RNA-guided endonuclease Cas9. Nature. 507, 62-67 (2014).

44. M. Shibata et al., Real-space and real-time dynamics of CRISPR-Cas9 visualized by highspeed atomic force microscopy. Nat Commun. 8, 1430 (2017).

45. E. K. Brinkman et al., Kinetics and Fidelity of the Repair of Cas9-Induced Double-Strand DNA Breaks. Mol. Cell. 70, 801-813.e6 (2018).

46. S. A. Verkuijl, M. G. Rots, The influence of eukaryotic chromatin state on CRISPR-Cas9 editing efficiencies. Curr. Opin. Biotechnol. 55, 68-73 (2018).

47. P. J. Skene, S. Henikoff, An efficient targeted nuclease strategy for high-resolution mapping of DNA binding sites. Elife. 6 (2017), doi:10.7554/eLife. 21856.

48. L. Deriano, D. B. Roth, Modernizing the nonhomologous end-joining repertoire: alternative and classical NHEJ share the stage. Annu. Rev. Genet. 47, 433-455 (2013).

49. M. Kosicki, K. Tomberg, A. Bradley, Repair of double-strand breaks induced by CRISPRCas9 leads to large deletions and complex rearrangements. Nat. Biotechnol. 36, 765-771

695 (2018). 